\title{
Conclusion du dossier
}

\author{
F. Joly $\cdot$ Y. Panis \\ (C) Lavoisier SAS 2016
}

Le syndrome de grêle court fait partie des maladies rares dont la prise en charge complexe nécessite une prise en charge multidisciplinaire très précoce dans des centres spécialisées. Le rôle du chirurgien est crucial et évolue tout au long de l'évolution de la maladie. Il est essentiel que des centres comprenant chirurgiens digestifs, gastroentérologues, nutritionnistes, réanimateurs, radiologues interventionnels, diététiciens et infirmiers de coordination, tous ayant une expertise dans le domaine de l'insuffisance intestinale, puissent être parfaitement identifiés et visibles pour améliorer le parcours de soins du patient. Ces cen- tres, aujourd'hui, sont souvent liés aux centres dits agréés de nutrition parentérale à domicile (NPAD). À l'avenir, il serait probablement nécessaire de rebaptiser ces centres comme centres de " réhabilitation intestinale pour maladies rares » où les patients pourront ainsi bénéficier d'une prise en charge en NPAD de qualité, mais aussi de la discussion des techniques chirurgicales de reconstruction, de la mise en place des facteurs trophiques intestinaux comme l'analogue de synthèse du GLP2 et d'une éducation thérapeutique pour améliorer l'autonomie du patient pour les soins. 